\title{
Shoe-shaped Interface for Inducing a Walking Cycle
}

\author{
Junji Watanabe*, Hideyuki Ando**, Taro Maeda** \\ * Graduate School of Information Science and Technology, The University of Tokyo \\ 7-3-1, Hongo, Bunkyo-ku, Tokyo, Japan \\ junji@star.t.u-tokyo.ac.jp \\ ** NTT Corp. Communication Science Laboratories \\ 3-1, Morinosato Wakamiya, Atsugi-shi, Kanagawa pref., Japan \\ hide,maeda@avg.brl.ntt.co.jp
}

\begin{abstract}
We propose a shoe-shaped interface designed to induce a specific walking cycle, and investigate stimulation techniques for effective induction of the walking cycle. The proposed interface is useful for walking navigation system, which enables the wearer's locomotion without paying attention to surrounding circumstances. The interface consists of a vibration motor for stimulation and pressure sensors for measuring walking cycle. Using sensory-motor synchronization of human body, this interface can induce a walking cycle effectively without mechanical constraint. The results of our experiments indicate that vibration stimuli at heel grounding timing enable effective induction and that wearers can smoothly shift their walking cycles to the indicated cycle, if the shift of the stimulation cycle is within the range of -100 and $+150 \mathrm{~ms}$ from their walking cycle.
\end{abstract}

Key words: Walk navigation, Shoe-shaped interface Sensory-motor synchronization

\section{Introduction}

When people walk, they have to alter the state of walking according to the change of the surrounding environment. When the traffic signal is changing to red, they will walk faster. When they get closer to bywalkers, the direction of walking will be changed. If appropriate velocity and direction of walking are indicated based on surrounding information, efficient locomotion can be performed. For example, we can approach the destination without stopping at red signal, and we can get to there at the expected time without getting nervous about the time. In addition to those, when several people use the navigation system at the same time, they can walk with comfortable distance. In order to realize this walk navigation system, it is important to control the direction and velocity of their walking. Authors have been studying on the direction induction system using vestibular stimulation [1][2]. Therefore, in this paper, we discuss velocity induction. Walking velocity is decided by step length and walking cycle, and the step length doesn't change even when the velocity changes. Consequently, we induced a walking cycle using a shoe-shaped interface (fig. 1) and investigated stimulation technique for effective induction.

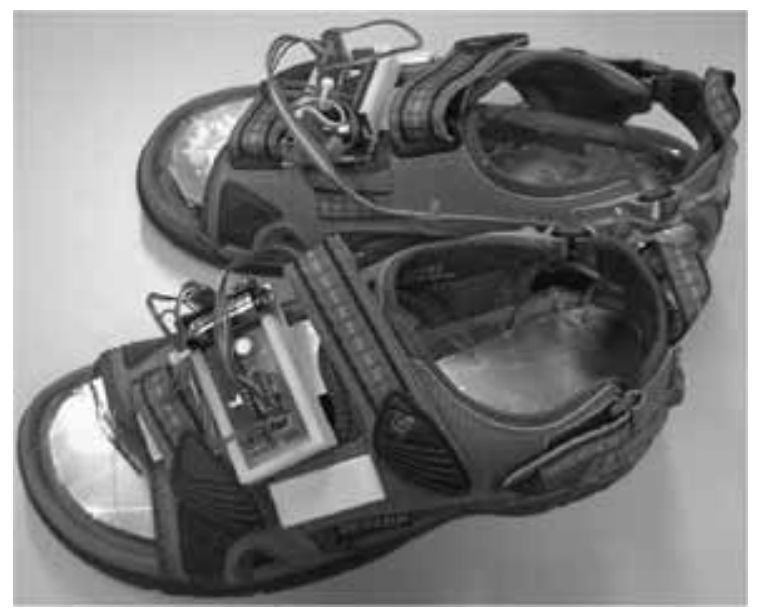

Fig. 1 Shoe-shaped interface

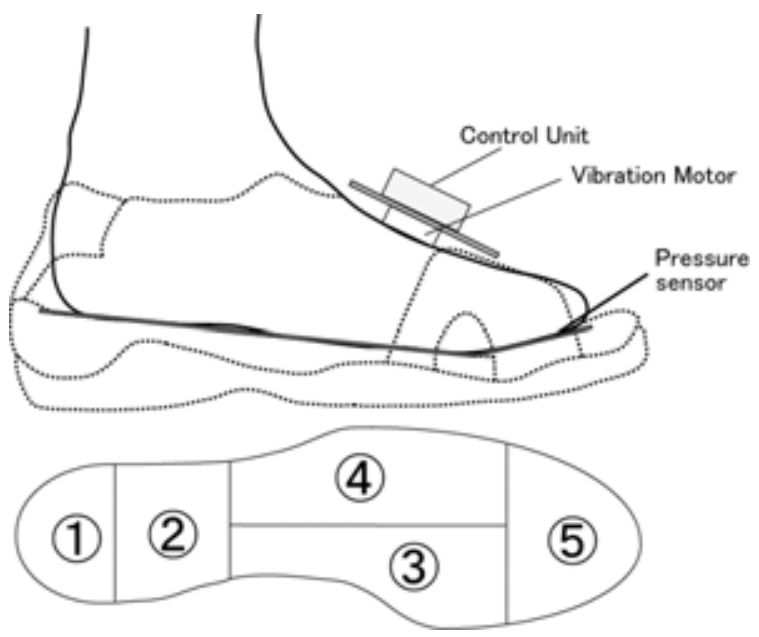

Fig. 2 Components of shoe-shaped interface

\section{Shoe-shaped interface}

\subsection{Sensory-motor synchronization}

When we indicate a specific walking cycle, it is difficult to directly indicate the cycle by an image or sounds, which represents "faster" or "slower". We, therefore, use sensory-motor synchronization for the induction method. The sensory-motor synchronization is that the rhythm of 
motor movement (walking) is unconsciously affected by sensory inputs and finally coincides with the rhythm of sensory input. For example, when we walk while hearing music, the walking cycle gradually coincides with the rhythm of the music. Using this sensory-motor synchronization of human body [4], this interface can induce a wearer's walking cycle effectively without mechanical constraint.

We used vibration stimuli for indicating a walking cycle, since, if visual or auditory stimuli were used, it could be inevitable to prevent wearers from getting information from surroundings. Consequently, we attached a small vibration motor on the instep of the shoe-shaped interface. The motor could give a $1.0 \mathrm{G}$ vibration for $200 \mathrm{~ms}$. Although this stimulus was presented on the shoes, wearer could perceive the vibration, and the vibration didn't interfere the walking. Pressure sensors were attached on the sole of the shoeshaped interface. Five pressure sensors were arranged on the sole of the interface as in fig. 2. We could measure the walking cycle and discriminate the state of walking by obtained pressure distribution. The measurement was performed at $100 \mathrm{~Hz}$.

\subsection{Characteristics of proposed system}

Several previous studies have been performed on walking stabilization based on sensory-motor synchronization [4-7]. In these studies, the walking cycle was measured with touch sensors on the sole, and sound stimuli for inducing a cycle were presented by a headphone. Using this system, they assisted aged or impaired person to maintain their stable walking. On the other hand, the walking navigation interface we are proposing is aimed to help able-bodied person perform effective locomotion. As a characteristic of this system, the sensors and stimulation device are implemented in one shoe-shaped interface.

We specified vibration timing in experiment 1 and the range of stimuli for effective navigation in experiment 2.

\section{Experiment 1}

\subsection{Apparatus and data analysis}

When we induced a specific walking cycle, the average walking cycle of the wearer was measured with pressure sensor attached on the sole of the interface. Then, the vibration whose cycle was in the vicinity of the averaged cycle, was presented, and the wearer walked in keep with the vibration cycle.

The pressure envelopes of the five sensors are shown in fig. 3. The horizontal and vertical axes represent time and measured pressure, respectively. The numbers in the figure corresponds the sensor numbers in fig. 2 below. When the sole lands, the pressure of the heel increases, then the pressure distribution shifts toward the toe. Using this pressure distribution, we can classify the states of walking into following five categories. Heel grounding, Sole Grounding, Grounding Middle, Heel Take off, and Toe Take off.

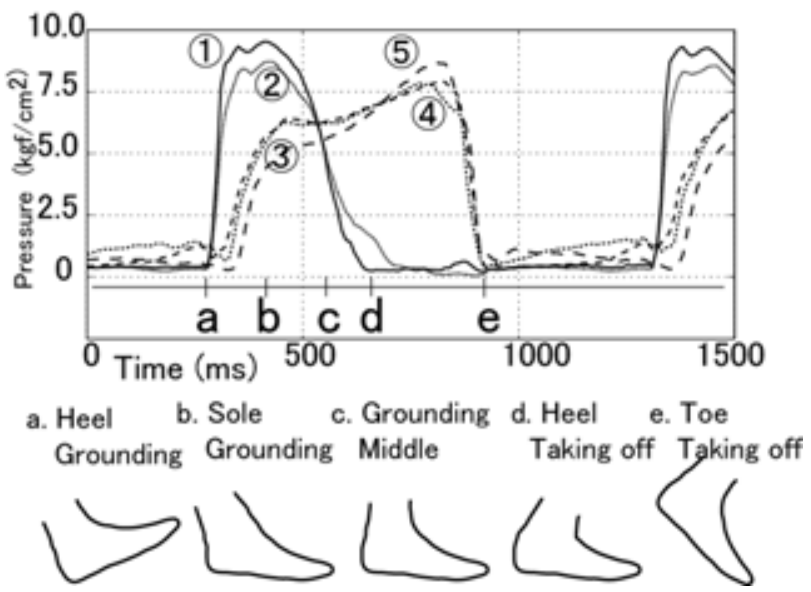

Fig. 3 Pressure distribution of plantar and state of walking

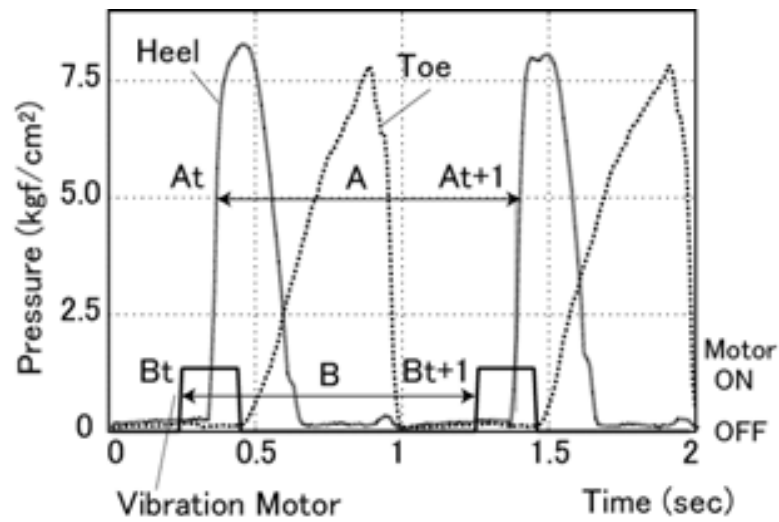

Fig. 4 Definition of walking cycle and vibration stimulation cycle

Figure 4 shows the definition of the walking and vibration cycle. The walking cycle is defined as the interval between the time At when the pressure of the heel (sensor number 1 ) exceeds $5 \mathrm{kgf} / \mathrm{cm} 2$ and the time $A t+1$ when the pressure of the heel exceeds $5 \mathrm{kgf} / \mathrm{cm} 2$ next time after At. The stimulation cycle is defined as the interval between the time $\boldsymbol{B} \boldsymbol{t}$ when the motor is turned on and the next stimulation time $\boldsymbol{B} \boldsymbol{t}+\mathbf{1}$. The phase difference $\varphi$ between waling and stimulation cycles is calculated by following formula.

$$
\varphi=2 \pi \times \frac{(A t-B t)}{B t+1-B t}
$$

Three men who had normal walking ability and whose ages were ranged from 24 to 29 , participated in this experiment.

\subsection{Procedure}

In this experiment, the subject walked in a large ground by 60 steps per one leg, about $120 \mathrm{~m}$. Experimental procedure was separated into following 2 
segment.

1, Measuring average walking cycle of the subject (Average measuring period :5-15 step)

2, Presenting vibration stimulus with the averaged cycle at one timing of the five walking state. (Stimulation period :16-60 step)

In the first segment, the average walking cycle was calculated during 10 steps (5-15 steps). In the second segment (16-60 steps), vibration stimulus with the averaged cycle started at one timing of the five walking state; Heel Grounding ( $0 \mathrm{~ms}$ after the pressure exceed the threshold), Sole Grounding (200ms after), Grounding Middle (400 ms after), Heel Taking off (600 $\mathrm{ms}$ after), Toe Taking off ( $800 \mathrm{~ms}$ after). Under this condition, 3 trials for each condition, total 15 trials were performed for each subject. The subjects were instructed simply, "to walk as synchronizing with stimulation cycle as exactly as possible". The subjects were not told that stimulation cycle coincided with average cycle.

This navigation technique uses unconscious sensorymotor synchronization of human body, and therefore, the induction can be performed without wearer's attention. Consequently, switch-push task was also imposed to the subjects, in order to distract wearer's conscious from the vibration stimulus. The subject walked, having a board, on which one red and blue LEDs were attached, and whose size was $100 \mathrm{~mm} \times 70 \mathrm{~mm}$. They were instructed to push red button, if a red LED was turned on, or to push blue button if a blue one was turned on, as soon as possible. LEDs were turned off when the button was pushed. One of the red or blue LED was turned on randomly with unrelated cycle to the walking cycle of the subjects, and the interval of the onset was randomized between $0.5-3.0 \mathrm{sec}$. This task was also effective to hinder the subject to confirm their current positions and velocities without shutting out visual information.

\subsection{Results}

The averaged walking cycles of three subjects were 1017, 1003, $1047 \mathrm{~ms}$, respectively. The phase difference $\varphi$ between walking cycle and vibration cycle, which is calculated based on formula 1 , is shown in fig. 5. All trials of one subject are described in this figure. The symbols " $a, b, c, d, e$ " represent the vibration timings of heel grounding, sole grounding, grounding middle, heel taking off and toe taking off, respectively. The tendency that phase difference converge to $0 \mathrm{rad}$ was observed. In other word, the subject adjusted the timing of vibration to the heel grounding timing. Although there were some trials converged to -2 pi rad, this means the heel grounding timing after one cycle. The same tendencies were observed also in the data of the other subjects. The

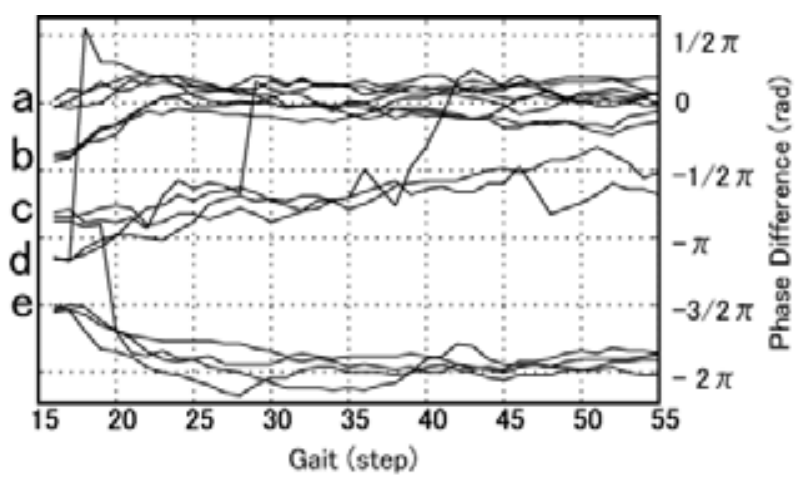

Fig. 5 Timing of stimulation for efficient induction

rate of heel grounding was $86.7 \%, 66.7 \%, 73.0 \%$. The result of this experiment indicates that subjects walked adjusting the timing of vibration to the heel grounding timing, when a stimulation, whose cycle was in the vicinity of their walking cycle, was presented. Therefore, the vibration stimulus was presented at the heel grounding timing in the next experiment.

\section{Experiment 2}

In experiment 2, we investigated the shift range of the vibration cycle, in which wearers could change their walking cycle to the given vibration cycle without psychological load. In this experiment, the subjects walked, while performing the same switch-push task as in exp. 1, and the vibration cycle was changed (increase or decrease) for inducing a walking cycle. We evaluated the change of psychological load by measuring reaction time of the switch-push task. If the subject gets psychological load when adjusting his walking cycle to the stimulation cycle, reaction time could increase. On the other hand, if the subject can change his walking cycle without psychological load, the reaction time will not change.

\subsection{Apparatus and procedure}

The same 3 subjects, who participated in the first experiment, performed this experiment. They walked 60 steps, about $120 \mathrm{~m}$. The same instruction and switchpush task as in exp. 1, were given for the subjects. The procedure of this experiment was classified into following 3 segments.

1, Measuring average walking cycle of the subject (Average measuring period :5-15 step)

2, Presenting vibration stimuli with the averaged cycle (Cycle synch period :16-35 step)

3, Presenting vibration stimuli with shifted cycle (Cycle shifted period : 36-60 step)

In the first segment, the average of the subject's walking cycle was calculated using 10 walking cycle (515 step). In the second segment (Cycle synch period :1635 step), vibration stimuli with the averaged cycle were 
presented at heel grounding timing. In the third segment (Cycle shifted period : 36-60 step), vibration stimuli with shifted cycle were presented. The shift of vibration cycle was varied between $-200,-150,-100,-50,0,50$, 100,150 and $200 \mathrm{~ms}$ from the averaged cycle. 3 trials for each 9 shift variation (27 trials), were performed for each subject. The reaction times of the switch-push task were also measured.

\subsection{Result}

When the amount of the shift was $+200 \mathrm{~ms}$, slow walking was observed, and it looked very difficult to coincide his walking cycle with the given stimulation cycle. When the amount of the shift was $-200 \mathrm{~ms}$, although it is possible to accord the cycles, his walking style became race walking. We evaluated psychological load of the subject using the reaction times of the switchpush task. Table 1 shows averages and standard deviations of the reaction times in the segment 2 and 3. The increase rate of averages and standard deviations are also shown.

The averages and standard deviations of reaction times in cycle synch period, are described in the first rows of each subjects' data in table 1 . They are about $300 \mathrm{~ms}$ and $100 \mathrm{~ms}$, respectively. These values indicate that switch-push task was certainly and stably performed. As for the values in cycle shifted period, described in the second rows of each subjects' data in table 1, although the average was equivalent to the value in the cycle synch period, the standard deviation shows different trend. Specifically the standard deviation become large, as the absolute amount of the shift rises.

In order to judge the difference between performances in the cycle synch and cycle shifted period, the increase rate is calculated by dividing the value in the cycle shifted period with the value in the synch period. If this rate is larger than 1.0, the performance of switch-push task was degraded by the change of the vibration cycle. If the rate is 1.0 , the change of vibration cycle didn't affect the task performance, say, the shift of walking cycle was performed without psychological load. The calculated rate are shown in the third rows of each subjects' data in table 1, and fig. 6. Horizontal and vertical axes in fig. 6 represent the amount of the cycle shift (ms) and the increase rate, respectively. The result of 3 subjects and averaged data of them are shown. The rates of averaged reaction times in fig 6(a) are about 1.0, although little increase is observed in the value of the shift $+200 \mathrm{~ms}$. On the other hand, the rates in fig. 6(b) increase when the amount of the shift is $-200 \mathrm{~ms}$, which induces fast walking, and greatly increase, when the shift is more than $+150 \mathrm{~ms}$. When we consider the conditions, in which the increase rates are less than 2.0, as the limit of stable walking, the wearer can adjust his walking cycle to the vibration cycle without psychological load within -150 and $+100 \mathrm{~ms}$.
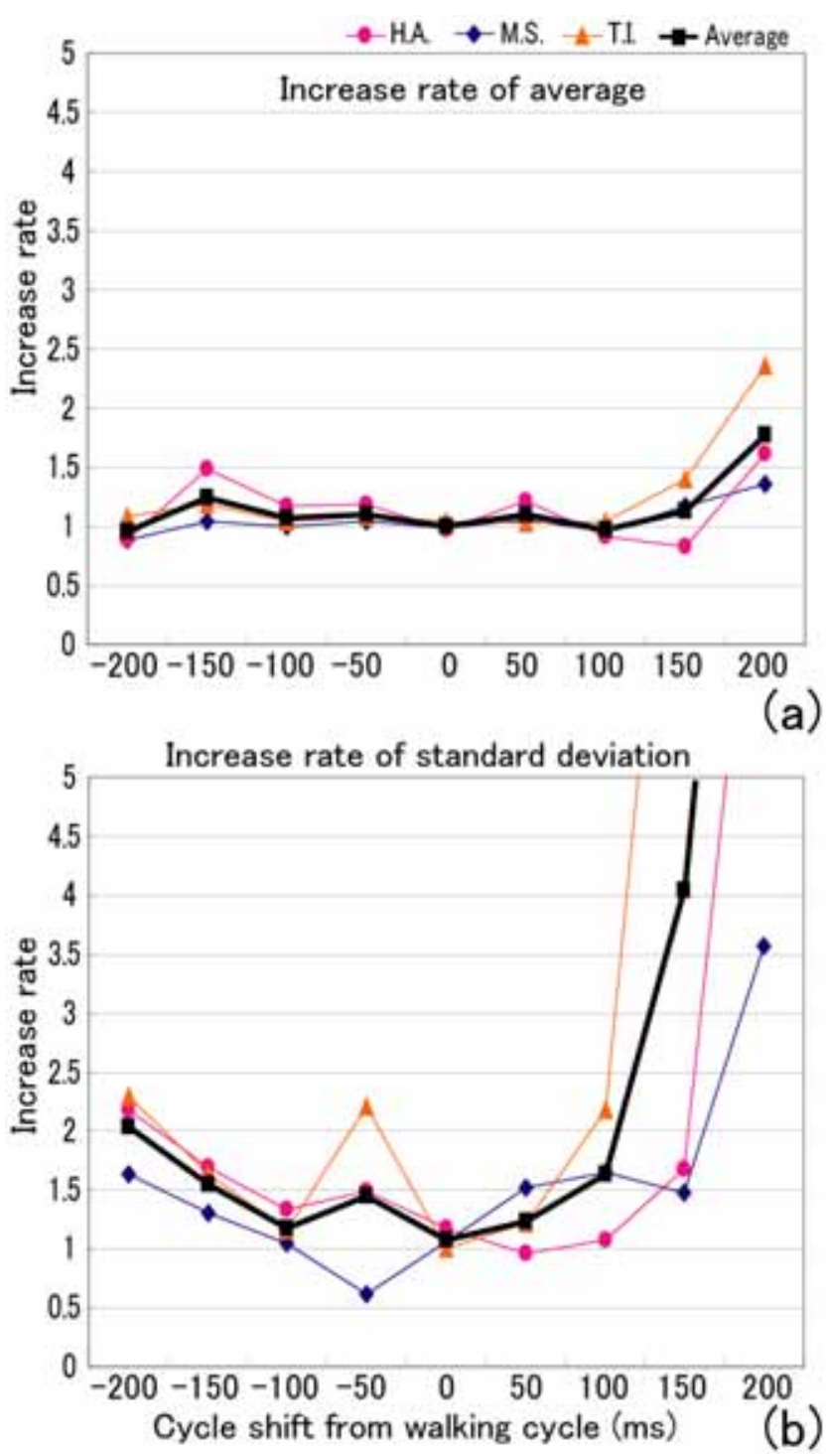

Fig. 6 Increase rate of (a)average (b)standard deviation

\section{Discussion}

The wearer was instructed to synchronize his walking cycle to the vibration cycle in our experiment, and it took about 5-10 sec to change his walking cycle (see fig. 2). In the Miyake's previous studies [4-7], they used cooperative induction and it required more than $10 \mathrm{sec}$ to realize stable walking. Our technique is suitable for controlling walking cycle due to its immediacy.

\section{Conclusion}

In current paper, we proposed a shoe-shaped interface for inducing a specific walking cycle, and investigated stimulation technique for effective induction The results of our experiments indicate that vibration stimuli at heel grounding timing enable effective induction and that the shift range between -100 to $+150 \mathrm{~ms}$ from wearer's walking cycle can lead his walking cycle to the stimuli's cycle without psychological load. 


\section{References}

1. Taro Maeda Hideyuki Ando, Maki Sugimoto, Junji Watanabe, and Takeshi Miki:"Wearable Robotics as a Behavioral Interface -The Study of the Parasitic Humanoid-", Proc of 6th International Symposium on Wearable Computers, pp.145-151 (2002).

2. Leah R. Benta, Bradford J. McFadyenb, Veronique French Merkleya, \%Paul M. Kennedya, J. Timothy Inglisa : "Magnitude effects of galvanic vestibular stimulation on the trajectory of human gait", Neuroscience Letters, Vol. 279, pp. 157-160 (2000).

3. E. Thelen, L.B. Smith: "A dynamic systems approach to the development of cognition and action", MIT Press (1998).

4. Y. Miyake, H. Shimizu:,"Mutual entrainment based human-robot communication field", Proc. of $3 \mathrm{rd}$.
IEEE Int. Workshop on Robot and Human Communication, pp. 118-123 (1994).

5. Y. Miyake, G. Taga, Y. Ohto, Y. Yamaguchi, H. Shimizu:"Mutual entrainment field based control in distributed autonomous robotic system", Proc. of 2nd. IEEE Int. Symp. on Distributed Autonomous Robotic Systems, pp. 85-88 (1994).

6. Y. Miyake, T. Miyagawa:"Internal observation and co-generative interface", Proc. of 1999 IEEE Int. Conf. on Systems, Man, and Cybernetics, pp. 229-237 (1999).

7. T. Muto, Y. Miyake:"Analysis of the Co-emergence Process on the Human-Human Cooperation", Proc. of 2002 IEEE Int. Conf. on Systems, Man, and Cybernetics, pp. MP1J2 (2002).

Table 1 Averages, standard deviations and their increase rate in the reaction time of switch-push task.

\begin{tabular}{|c|c|c|c|c|c|c|c|c|c|}
\hline Amount of shift (ms) & -200 & -150 & -100 & -50 & 0 & +50 & +100 & +150 & +200 \\
\hline \multirow{10}{*}{$\begin{array}{l}\text { Average reaction time in phase } \\
\text { synchronizing period, and phase } \\
\text { shifted period and ratio of them. } \\
\text { Data of } 3 \text { subjects (HA, MS, TI) } \\
\text { are sequentially presented. }\end{array}$} & \multicolumn{9}{|c|}{ Average of Reaction time (ms) } \\
\hline & 301.3 & 380.3 & 313.1 & 294.3 & 288.3 & 289.5 & 258.5 & 257.9 & 374.9 \\
\hline & 487.8 & 314.3 & 285.6 & 355.6 & 278.3 & 343.3 & 303.4 & 381.5 & 334.5 \\
\hline & 1.62 & 0.83 & 0.91 & 1.21 & 0.97 & 1.19 & 1.17 & 1.48 & 0.89 \\
\hline & 311.2 & 320.7 & 354.1 & 292.3 & 282.1 & 312.7 & 302.0 & 269.1 & 374.6 \\
\hline & 422.8 & 374.2 & 340.5 & 309.7 & 278.0 & 325.0 & 303.4 & 282.2 & 333.0 \\
\hline & 1.36 & 1.17 & 0.96 & 1.06 & 0.98 & 1.04 & 1.00 & 1.05 & 0.89 \\
\hline & 341.5 & 298.3 & 301.9 & 301.8 & 304.4 & 332.5 & 297.7 & 331.3 & 299.1 \\
\hline & 803.3 & 418.1 & 313.3 & 311.3 & 318.5 & 362.5 & 311.2 & 392.7 & 323.4 \\
\hline & 2.35 & 1.40 & 1.04 & 1.03 & 1.05 & 1.09 & 1.05 & 1.19 & 1.08 \\
\hline \multirow{10}{*}{$\begin{array}{l}\text { Standard deviation of reaction } \\
\text { time in phase synchronizing } \\
\text { period, and phase shifted period } \\
\text { and ratio of them. } \\
\text { Data of } 3 \text { subjects (HA, MS, TI) } \\
\text { are sequentially presented. }\end{array}$} & \multicolumn{9}{|c|}{ Standard Deviation of Reaction time (ms) } \\
\hline & 59.1 & 69.4 & 67.8 & 81.8 & 73.2 & 58.1 & 68.3 & 67.6 & 85.3 \\
\hline & 470.5 & 115.9 & 72.5 & 79.1 & 85.7 & 86.5 & 91.1 & 114.2 & 185.6 \\
\hline & 7.96 & 1.67 & 1.07 & 0.97 & 1.17 & 1.49 & 1.33 & 1.69 & 2.18 \\
\hline & 84.2 & 86.3 & 75.1 & 58.1 & 76.1 & 85.2 & 87.3 & 66.3 & 86.8 \\
\hline & 300.4 & 127.8 & 123.4 & 88.4 & 80.6 & 53.0 & 91.1 & 86.5 & 142.0 \\
\hline & 3.57 & 1.48 & 1.64 & 1.52 & 1.06 & 0.62 & 1.04 & 1.30 & 1.64 \\
\hline & 60.8 & 72.0 & 54.8 & 68.7 & 81.9 & 86.0 & 59.3 & 78.2 & 76.8 \\
\hline & 892.6 & 644.6 & 119.2 & 84.1 & 82.0 & 189.9 & 69.1 & 127.8 & 175.7 \\
\hline & 14.68 & 8.96 & 2.17 & 1.22 & 1.00 & 2.21 & 1.16 & 1.63 & 2.29 \\
\hline
\end{tabular}

